\title{
Os fatores de risco da infecção do trato urinário na gravidez: revisão integrativa
}

\section{The risk factors of urinary tract infection in pregnancy: an integrative review}

Flávia Fragoso dos Santos Fioravante ${ }^{1} \bullet$ Gisella de Carvalho Queluci ${ }^{2}$

\section{RESUMO}

Objetivos: Realizar uma revisão integrativa para analisar os fatores de risco referentes à ocorrência da infecção urinária na gravidez. Método: Revisão integrativa da literatura realizada nas bases LILACS,PAHO,WHOLYS,BIBLIOTECA COCHRANE, MEDLINE e CINAHL, no período de 01 de abril de 2015 a 01 de maio de 2015, baseada na seguinte questão de pesquisa: no que se refere à prevenção de agravos, quais são os fatores de risco relacionados com a infecção urinária na gravidez? Resultados: Dez artigos foram selecionados, e evidenciaram-se como fatores de risco para a ocorrência da infecção urinária na gravidez: baixa escolaridade, baixo nível socioeconômico, atividade sexual, história prévia de infecção urinária, anemia materna, adolescência, terceiro trimestre de gravidez, multiparidade, atraso no esvaziamento da bexiga, não lavar a genitália pré e pós-coito, e conviver com cães e gatos. Conclusões: $\mathrm{A}$ análise dos fatores de risco relacionados com a infecção urinária na gravidez poderá trazer benefícios para a saúde materna e fetal bem como contribuir com uma atuação profissional mais qualificada.

Palavras-chave: Fatores de risco; Infecções urinárias; Gravidez.

\section{ABSTRACT}

Objective: Conduct an integrative review to analyze the risk factors related to the occurrence of urinary infection in pregnancy. Method: Integrative literature review conducted in LILACS, PAHO, WHOLYS, COCHRANE LIBRARY, MEDLINE and CINAHL, in the period from April 1, 2015 the May 1, 2015, and based on the following research question: with regard to prevention aggravations, what are the risk factors associated with urinary infection in pregnancy. Results: Ten articles were selected, and it was shown to be risk factors for the occurrence of urinary tract infection in pregnancy: low education, low socioeconomic status, sexual activity, history of urinary tract infection, maternal anemia, adolescence, third trimester of pregnancy, multiparity, delayed emptying of the bladder, do not wash the genitals pre and post-coital, and live with dogs and cats. Conclusions: The analysis of risk factors related to urinary tract infection in pregnancy can bring benefits for maternal and fetal health and contribute to a more qualified professional performance.

Keywords: Risk factors; Urinary tract infections; Pregnancy.

1. Enfermeira, Mestranda, Programa de Mestrado Profissional em Enfermagem Assistencial (MPEA), Escola de Enfermagem Aurora de Afonso Costa (EEAAC), Universidade Federal Fluminense (UFF), Niterói, RJ, Brasil. E-mail: flaviafragoso@hotmail.com

2. Enfermeira, Doutora em Enfermagem, Escola de Enfermagem Anna Nery (EEAN), Universidade Federal do Rio de Janeiro (UFRJ), Professora Adjunta em Enfermagem, Escola de Enfermagem Aurora de Afonso Costa (EEAAC), Universidade Federal Fluminense (UFF), Docente Permanente do Mestrado Profissional em Enfermagem Assistencial (MPEA), Escola de Enfermagem Aurora de Afonso Costa (EEAAC), Universidade Federal Fluminense (UFF), Niterói, RJ, Brasil. E-mail: gisellaqueluci@yahoo.com.br

Os autores declaram não haver conflitos de interesse nem fontes de financiamento. 


\section{INTRODUÇÃO}

A infecção do trato urinário (ITU) constitui uma das principais afecções que acometem a mulher no ciclo gravídico. É caracterizada pela colonização, invasão e multiplicação de agentes infecciosos no sistema urinário e tem como principal agente etiológico a Escherichia coli, que é uma bactéria gram negativa comumente encontrada colonizando o trato intestinal ${ }^{1-2}$. A esse tipo de infecção associam-se tanto complicações maternas, como anemia, pré-eclampsia, eclampsia, corioamnionite e endometrite, quanto perinatais, como prematuridade, baixo peso ao nascer $(<2.500 \mathrm{~g})$, restrição de crescimento intraútero, além do óbito neonatal ${ }^{3}$.

Vale ressaltar que a prematuridade, no Brasil, é um dos principais fatores que contribuem para a mortalidade infantil que, em 2011, foi de 15,3/1000 nascidos vivos, considerada alta quando comparada a países com desenvolvimento econômico semelhantes $(<10 / 1000$ nascidos vivos). E que mais de $60 \%$ dos óbitos ocorridos em menores de 1 ano, encontram-se no período neonatal (10,6/1000 nascidos vivos), tendo como principal causa de morte na primeira semana de vida, a prematuridade e o baixo peso ao nascer ${ }^{4}$.

Tais complicações são consideradas, em sua maioria, evitáveis. Isto se dá através de uma assistência de prénatal integral e resolutiva que atue na promoção à saúde, prevenção de agravos, tratamento adequado e recuperação da saúde. A identificação precoce dos fatores de risco, nesse caso, para a ITU durante a gravidez, e o seu manejo adequado, contribuiriam para a redução das complicações materno-fetais e para uma prática profissional de saúde mais qualificada.

Nesse sentido, esse estudo teve como objetivo realizar uma revisão integrativa para analisar os fatores de risco referentes à ocorrência da infecção urinária na gravidez.

\section{MÉTODO}

Trata-se de uma revisão integrativa da literatura, a qual se constitui em uma ferramenta de natureza complexa, possibilitando ao pesquisador a síntese de achados de diferentes pesquisas sobre uma mesma temática ${ }^{5}$.

Para a elaboração da questão de pesquisa foi aplicada a estratégia PIO, uma adaptação da PICO, já que não houve um grupo-controle para comparação, sendo assim, o C não foi utilizado. Nesse caso, o P constituiu-se nas mulheres grávidas, o I nas infecções urinárias e o $O$ nos fatores de risco. A partir disso, construiu-se a seguinte questão de pesquisa: No que se refere à prevenção de agravos, quais são os fatores de risco para a ocorrência da infecção urinária na gravidez?

$\mathrm{Na}$ estratégia de busca, foi realizado um levantamento dos estudos indexados nos seguintes Portais: Biblioteca Virtual em Saúde (BVS), PubMed e Cinahl. Na BVS, as bases pesquisadas foram: LILACS, Paho, Wholys e Biblioteca Cochrane. E, na PubMed, Medline.
O recorte temporal da pesquisa foi estendido para os últimos 10 anos (2005-2014), em virtude do número reduzido de publicações atuais encontradas. A coleta de dados ocorreu no período de 01 de abril de 2015 a 01 de maio de 2015.

Os critérios de inclusão foram os artigos eletrônicos, teses e dissertações, publicados em português, inglês e espanhol, provenientes de estudos primários e que estavam de acordo com a questão de pesquisa. Já os critérios de exclusão foram os estudos secundários, as recomendações de especialistas ou de associações de classe e os estudos sem metodologia clara.

Os descritores controlados utilizados foram baseados nos Descritores em Ciências da Saúde (DeCS) e nos termos Medical Subject Headings (Mesh). Foram eles: fatores de risco, gravidez e infecções urinárias utilizados na BVS, e, risk factors, pregnancy e urinary tract infections utilizados na Medline. Na Cinahl, foram utilizados os descritores risk factors, pregnancy e urinary tract infections.

$\mathrm{Na}$ BVS, a sintaxe utilizada continha tanto descritores controlados quanto palavras-chave observados a seguir: gravidez OR gestante AND infecções urinárias AND fatores de risco. Na Medline, a sintaxe configurou-se em ("pregnancy"[MeSH Terms] OR "pregnancy"[All Fields]) AND ("urinary tract infections"[MeSH Terms] OR ("urinary"[All Fields] AND "tract"[All Fields] AND "infections"[All Fields]) OR "urinary tract infections"[All Fields]) AND ("risk factors"[MeSH Terms] OR ("risk"[All Fields] AND "factors"[All Fields]) OR “risk factors"[All Fields]). E, na CINAHL, a sintaxe utilizada foi pregnancy AND urinary tract infections AND risk factors.

O fluxo de seleção dos estudos realizado por 2 revisores ocorreu com base nas seguintes etapas: leitura dos títulos e resumos, seleção dos estudos de acordo com a questão de pesquisa e com os critérios de inclusão e exclusão, leitura dos textos completos, novamente a seleção dos estudos de acordo com a questão de pesquisa e com os critérios de inclusão e exclusão, preenchimento das fichas de descrição dos estudos e avaliação da qualidade dos estudos, com base na classificação do nível de evidência de Kysas e Hood ${ }^{6-7}$. Após um consenso entre os revisores, foram obtidas 10 publicações que atendiam às questões do estudo.

Esses estudos foram identificados de acordo com as variáveis referentes ao título, ano, país, tipo de estudo e nível de evidência de Kysas e Hood.

A Prática Baseada em Evidências vem preencher a lacuna que existe entre a prática e a teoria. Para tanto, busca a resolução dos problemas encontrados na prática através das melhores evidências. Devido a isso, classifica os tipos de estudo quanto à melhor evidência.

Baseando-se na classificação de Kysas e $\mathrm{Hood}^{6-7}$, a melhor evidência está no nível 10, que inclui os estudos de revisões sistemáticas com metanálise de ensaios clínicos randomizados; em seguida, o nível 9, com revisões 


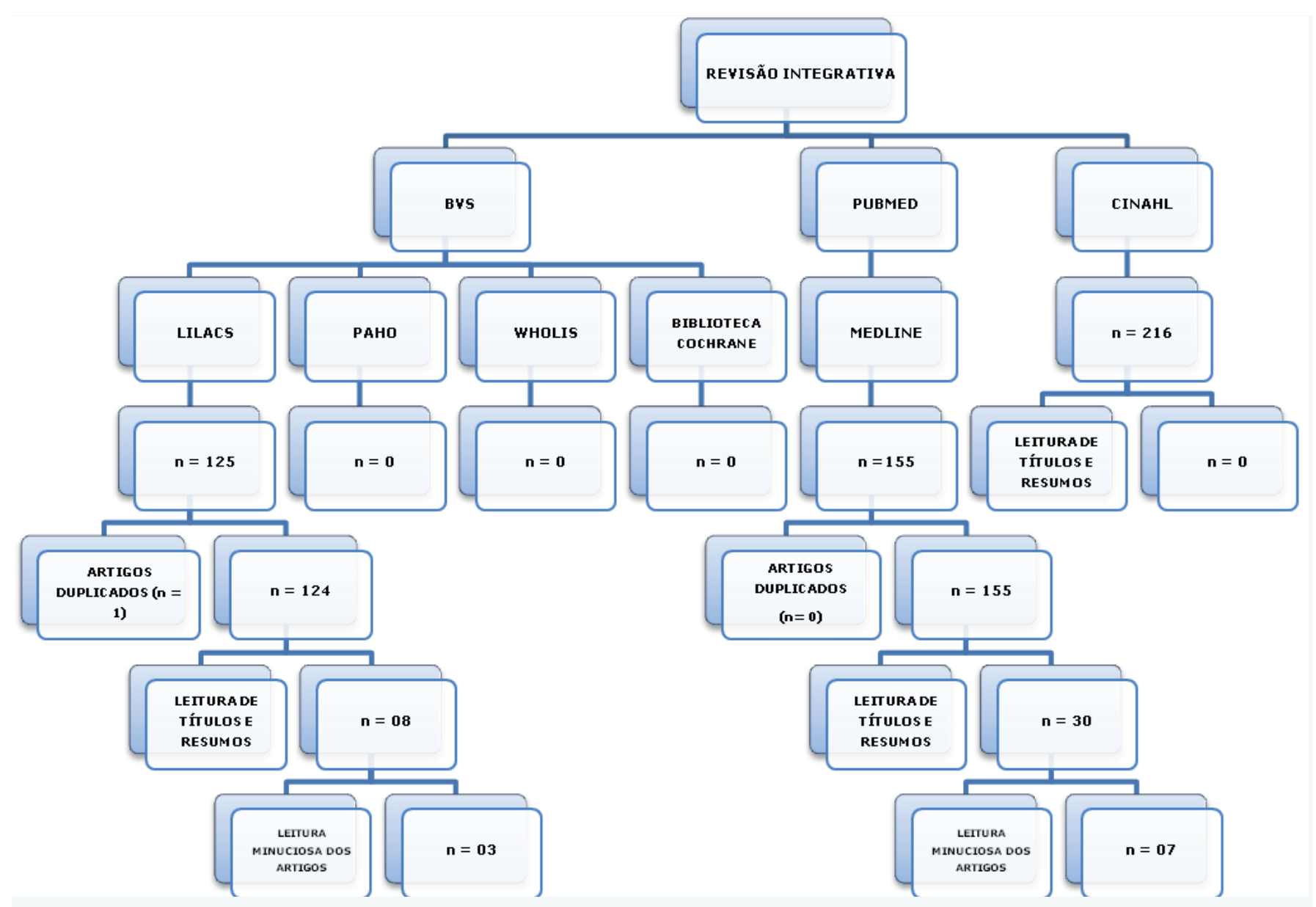

Figura 1. Fluxograma da revisão integrativa da literatura científica, elaborado pelos autores.

sistemáticas com metanálise; nível 8, com ensaios clínicos randomizados; nível 7 , com guias de prática clínica; nível 6 , com estudos de coorte e caso-controle; nível 5 , com estudos observacionais (longitudinais ou transversais); nível 4 , com casos clínicos e série de casos clínicos e série de casos; nível 3, com pesquisa básica laboratorial; nível 2 , com opiniões de especialistas; e o nível 1, com as revisões não sistemáticas da literatura, este considerado o menor nível de evidência científica.

\section{RESULTADOS}

A amostra compreendeu 10 estudos, conforme o fluxograma da figura 1. E, a partir da análise desses estudos, quanto a origem, verificou-se que, das 10 publicações, 07 eram internacionais e 03 eram nacionais. As publicações internacionais $(n=7)$ foram provenientes da Índia, Etiópia, Dinamarca, Sudão, Paquistão, Irã e Tailândia, enquanto as nacionais $(n=3)$ foram do Rio de Janeiro, Rio Grande do Sul e Paraná. De acordo com o tipo de publicação, todos eram artigos científicos eletrônicos $(n=10)$.

Quanto ao tipo de estudo, 8 eram do tipo transversal, 1 coorte e 1 caso-controle. Portanto, 8 estudos apresentaram o nível 5 de evidência, enquanto 2 apresentaram o nível 6. $E$, no que diz respeito à temática dos estudos, os 10 evidenciaram os fatores de risco para a ocorrência da infecção urinária na gravidez.
A partir dos principais resultados encontrados nos estudos acima, identificou-se os fatores de risco relacionados com a ocorrência da infecção urinária durante a gestação.

\section{DISCUSSÃO}

As publicações encontradas são, na sua maioria, internacionais, pertencentes à área médica e provenientes de países onde grande parte da população é de baixa condição socioeconômica, como a Índia, o Paquistão, o Sudão, o Irã, a Tailândia e a Etiópia.

Nesse estudo de revisão, $80 \%$ das publicações utilizaram a metodologia do estudo transversal, que, segundo a classificação de Kysas e Hood, encontram-se no nível 5 de evidência. $O$ estudo transversal é coerente com o que se objetivou pesquisar, isto é, os fatores de risco para a ocorrência da infecção urinária durante a gravidez.

Os fatores de risco evidenciados na tabela 3 constituíram-se em baixa escolaridade, baixo nível socioeconômico, atividade sexual, história prévia de infecção urinária, anemia materna, adolescência, diabetes, multiparidade, terceiro trimestre de gravidez, atraso no esvaziamento da bexiga, não lavar a genitália pré e póscoito, e conviver com cães e gatos.

A baixa renda familiar e a baixa escolaridade são características socioeconômicas que podem afetar a saúde do 
Tabela 1 - Identificação das publicações quanto ao título, revista, ano, país, método e nível de evidência. (n=10). Niterói, 2015.

\begin{tabular}{|c|c|c|c|c|}
\hline Título/Revista & Ano & País & Tipo de Estudo & $\begin{array}{l}\text { Nível de } \\
\text { Evidência }\end{array}$ \\
\hline $\begin{array}{l}\text { 1- Diagnosis of asymptomatic bacteriuria and associated risk } \\
\text { factors among pregnant women in Mangalore, Karnataka, India }{ }^{8} / \\
\text { Journal of Clinical and Diagnostic Research }\end{array}$ & 2014 & Îndia & $\begin{array}{l}\text { Estudo } \\
\text { Transversal }\end{array}$ & 5 \\
\hline $\begin{array}{l}\text { 2- Assessment of urinary infection management during prenatal } \\
\text { care in pregnant women attending public health care units in the } \\
\text { city of Rio de Janeiro, Brazil }{ }^{9} \text { / Revista Brasileira de Epidemiologia }\end{array}$ & 2013 & Brasil & $\begin{array}{l}\text { Estudo } \\
\text { Transversal }\end{array}$ & 5 \\
\hline $\begin{array}{l}\text { 3- Associated risk factors of urinary tract infection among } \\
\text { pregnant women at Felege Hiwot Referral Hospital, Bahir Dar, } \\
\text { North West Ethiopia }{ }^{10} / \text { BioMed Central Research Notes }\end{array}$ & 2013 & Etiópia & $\begin{array}{l}\text { Estudo } \\
\text { Transversal }\end{array}$ & 5 \\
\hline $\begin{array}{l}\text { 4- Prevalência e fatores associados à internação hospitalar para } \\
\text { tratamento da infecção do trato urinário durante a gestação }{ }^{11} / \\
\text { Revista Brasileira de Ginecologia e Obstetrícia }\end{array}$ & 2013 & Brasil & $\begin{array}{l}\text { Estudo } \\
\text { Transversal }\end{array}$ & 5 \\
\hline $\begin{array}{l}\text { 5- Living with cat and dog increases vaginal colonization with } \mathrm{E} \text {. } \\
\text { coli in pregnant women }{ }^{12} / \text { PLOS ONE }\end{array}$ & 2012 & Dinamarca & Estudo de Coorte & 6 \\
\hline $\begin{array}{l}\text { 6- Epidemiology of urinary tract infections and antibiotics } \\
\text { sensitivity among pregnant women at Khartoum North Hospital }{ }^{13} \\
\text { / Journal of Paquistan Medical Association }\end{array}$ & 2011 & Sudão & $\begin{array}{l}\text { Estudo } \\
\text { Transversal }\end{array}$ & 5 \\
\hline $\begin{array}{l}\text { 7- Risk factors of urinary tract infection in pregnancy }{ }^{14} / \text { Annals } \\
\text { of Clinical Microbiology and Antimicrobials }\end{array}$ & 2010 & Paquistão & $\begin{array}{l}\text { Estudo } \\
\text { Transversal }\end{array}$ & 5 \\
\hline $\begin{array}{l}\text { 8- Avaliação de infecção urinária em gestantes do município } \\
\text { de Marechal Cândido Rondom - } \mathrm{PR}^{15} / \text { Arquivos de Ciências da } \\
\text { Saúde da UNIPAR }\end{array}$ & 2010 & Brasil & $\begin{array}{l}\text { Estudo } \\
\text { Transversal }\end{array}$ & 5 \\
\hline $\begin{array}{l}\text { 9- Hygiene practices and sexual activity associated with urinary } \\
\text { tract infection in pregnant women }{ }^{16} / \text { La Revue de Santé de la } \\
\text { Méditerranée Orientale }\end{array}$ & 2009 & Irã & $\begin{array}{l}\text { Estudo de } \\
\text { Caso-Controle }\end{array}$ & 6 \\
\hline $\begin{array}{l}\text { 10- Risk factors related to asymptomatic bacteriúria in pregnant } \\
\text { women }^{17} / \text { Journal of the Medical Association of Thailand }\end{array}$ & 2009 & Tailândia & $\begin{array}{l}\text { Estudo } \\
\text { Transversal }\end{array}$ & 5 \\
\hline Total & 10 & & & \\
\hline
\end{tabular}

Tabela 2 - Título das publicações e seus principais achados. Niterói, RJ, 2015.

\section{Título das Publicações}

1- Diagnosis of asymptomatic bacteriuria and associated risk factors among pregnant women in Mangalore, Karnataka, India ${ }^{8}$

2- Assessment of urinary infection management during prenatal care in pregnant women attending public health care units in the city of Rio de Janeiro, Brazil ${ }^{9}$

3- Associated risk factors of urinary tract infection among pregnant women at Felege Hiwot Referral Hospital, Bahir Dar, North West Ethiopia ${ }^{10}$

4- Prevalência e fatores associados à internação hospitalar para tratamento da infecção do trato urinário durante a gestação ${ }^{11}$

5- Living with cat and dog increases vaginal colonization with $\mathrm{E}$. coli in pregnant women ${ }^{12}$

\section{Principais Achados}

A prevalência da bacteriúria assintomática em mulheres grávidas foi 13,2\%. Destas, 15\% ocorreram em mulheres grávidas com baixo nível socioeconômico.

Os fatores de risco encontrados para o manejo inadequado da ITU pelos profissionais de saúde durante o pré-natal em relação às gestantes foram a cor parda, a menor escolaridade e o estado nutricional eutrófico. E, os fatores de risco para a ocorrência da ITU na gravidez foram a adolescência, o diabetes e a anemia.

Os fatores fortemente associados a ITU se constituíram em baixa renda familiar, atividade sexual igual ou maior a três vezes por semana, anemia materna e história prévia de ITU. E, os de baixa associação, em idade materna entre 25 e 34 anos e anormalidade geniturinária.

A prevalência da ITU na gestação foi de 2,9\%, sendo maior nas mulheres com menor nível econômico, mais jovens (<20 anos) e com menor escolaridade (ensino fundamental).

O baixo peso ao nascer e o trabalho de parto pré-termo foram os desfechos encontrados.

Mulheres grávidas que conviviam com cães e gatos, apresentaram uma colonização vaginal por Escherichia coli igual a 15\%, enquanto as mulheres que não conviviam com esses animais apresentaram uma colonização vaginal de $8 \%$. E $32 \%$ das mulheres que conviveram com cães e gatos apresentaram infecção urinária durante a gravidez, enquanto as que não conviveram com esses animais, apresentaram $21 \%$. 
Tabela 2 - continuação

\begin{tabular}{l|l}
\hline \multicolumn{1}{c|}{ Título das Publicações } & \multicolumn{1}{c}{ Principais Achados } \\
\hline $\begin{array}{l}\text { 6- Epidemiology of urinary tract infections } \\
\text { and antibiotics sensitivity among pregnant } \\
\text { women at Khartoum North Hospital }{ }^{13}\end{array}$ & $\begin{array}{l}\text { A prevalência de mulheres grávidas na ITU foi igual a 14\%. A idade materna, o índice } \\
\text { de massa corporal e a idade gestacional não foram achados como fatores de risco } \\
\text { para ITU na gravidez. A principal bactéria isolada foi a Escherichia coli (42,4\%), que } \\
\text { apresentou resistência a amoxicilina, ácido naladixic, nitrofurantoína, ciprofloxacina } \\
\text { cotrimoxazol, amoxicilina / clavulanato e norfloxacina. }\end{array}$ \\
\hline $\begin{array}{l}\text { 7- Risk factors of urinary tract infection in } \\
\text { pregnancy }{ }^{14}\end{array}$ & $\begin{array}{l}\text { Os fatores de risco associados à infecção urinária na gravidez foram a baixa } \\
\text { escolaridade, a atividade sexual, a história prévia de ITU, a multiparidade e a baixa } \\
\text { condição sócio-econômica. }\end{array}$ \\
\hline $\begin{array}{l}\text { 8- Avaliação de infecção urinária em } \\
\text { gestantes do município de Marechal Cândido } \\
\text { Rondom - PR }{ }^{15}\end{array}$ & $\begin{array}{l}\text { A prevalência da ITU entre as gestantes foi de 56\%, sendo a Escherichia coli (63,1\%) } \\
\text { o principal agente causador. As gestantes no 30 trimestre apresentaram maior } \\
\text { incidência da infecção urinária (71,4\%). }\end{array}$ \\
\hline $\begin{array}{l}\text { 9- Hygiene practices and sexual activity } \\
\text { associated with urinary tract infection in } \\
\text { pregnant women }{ }^{16}\end{array}$ & $\begin{array}{l}\text { Os fatores encontrados associados à ITU na gravidez foram o não urinar após o } \\
\text { coito, o não lavar a genitália pré e pós-coito, o atraso no esvaziamento voluntário } \\
\text { da bexiga e o intercurso sexual igual ou maior a três vezes por semana. }\end{array}$ \\
\hline $\begin{array}{l}\text { 10- Risk factors related to asymptomatic } \\
\text { bacteriúria in pregnant women }{ }^{17}\end{array}$ & $\begin{array}{l}\text { Identificou-se somente o baixo nível educacional como um fator de risco } \\
\text { significante para a bacteriúria assintomática em mulheres grávidas. }\end{array}$ \\
\hline
\end{tabular}

Quadro 3- Fatores de risco de maior associação com a infecção urinária na gravidez, de acordo com cada publicação. Niterói, RJ, 2015.

\begin{tabular}{|l|c|c|}
\hline \multicolumn{1}{|c|}{ Fatores de Risco } & Publicação & N \\
\hline Baixa escolaridade & $1 ; 4 ; 7 ; 10$ & 04 \\
Baixo nível socioeconômico & $1 ; 3 ; 4 ; 7$ & 04 \\
Atividade Sexual & $3 ; 7 ; 9$ & 03 \\
História prévia de infecção urinária & $1 ; 3 ; 7$ & 03 \\
Anemia materna & $1 ; 2 ; 3$ & 02 \\
Adolescência & $2 ; 4$ & 02 \\
Diabetes & $1 ; 2$ & 02 \\
Multiparidade & $1 ; 7$ & 02 \\
Terceiro trimestre de gravidez & 8 & 01 \\
Atraso no esvaziamento da bexiga & 9 & 01 \\
Não lavar a genitália pré e pós-coito & 9 & 01 \\
Não urinar após o coito & 9 & 01 \\
Conviver com cães e gatos & 5 & 01 \\
\hline
\end{tabular}

indivíduo. No que diz respeito à ITU na gravidez, estas podem associar-se a questões de déficit de higiene e nutrição, os quais podem propiciar o aparecimento de infecções ${ }^{18-20}$.

A atividade sexual, o não lavar a genitália pré e póscoito e o não urinar após o coito predispõem à ocorrência da infecção urinária, já que o uropatógeno mais comum é a Escherichia coli, frequentemente encontrado no intestino e na região perineal. Durante o coito, o risco de contaminação da uretra por esse patógeno é aumentado ${ }^{19} .0$ ato de urinar após o coito ajuda a eliminar os patógenos que possam ter migrado para a uretra.

As características obstétricas como multiparidade e último trimestre de gravidez são também fatores de riscos para a ITU na gravidez evidenciadas em outros estudos ${ }^{20-22}$. Isto se deve, principalmente, às mudanças ocorridas no corpo feminino durante a gravidez, no qual se observa, principalmente no último trimestre, o efeito do peso de um útero maior sobre o ureter e ao maior relaxamento da musculatura da bexiga, ocasionando a estase urinária. Esta, por sua vez, favorece a proliferação de microorganismos ${ }^{19}$.
Por isso, o atraso no esvaziamento voluntário da bexiga também é prejudicial à saúde.

Quanto à idade, as gestantes mais jovens, principalmente as adolescentes, foram as que apresentaram maior risco para a infecção urinária. Em um estudo realizado na Paraíba, com 100 gestantes acompanhadas no Sistema Único de Saúde, também se observou uma maior prevalência da infecção urinária nas mulheres mais jovens ${ }^{18}$. Isto pode estar relacionado ao fato de, frequentemente, as mulheres mais jovens possuírem uma vida sexual mais ativa.

Patologias maternas como anemia, diabetes e história prévia de ITU, também foram associadas à infecção urinária em outros estudos ${ }^{23}$. Em um estudo realizado com gestantes diabéticas e não diabéticas, verificou-se um aumento de 2,5 vezes da ocorrência de bacteriúria assintomática nas que possuíam diabetes ${ }^{24}$.

Outro achado importante foi a maior prevalência da infecção urinária em gestantes que convivem com cães e gatos ${ }^{12}$. Neste estudo, foi levantada a suposição de que animais de estimação podem ser importantes transmissores de patógenos para os seus proprietários, a longo prazo. Isto foi documentado em outros estudos, os quais relataram a contaminação intradomiciliar por Escherichia coli entre humanos e animais de estimação $25-26$.

Nesse sentido, a identificação precoce de todos esses fatores de risco acima mencionados no cuidado à gestante possibilitará intervenções profissionais e resultados mais efetivos na assistência de enfermagem, visto que o mesmo estará pautado nas melhores evidências científicas encontradas ${ }^{27}$.

\section{CONCLUSÕES}

A análise dos artigos encontrados na revisão integrativa da literatura científica possibilitou evidenciar os principais fatores de risco referentes à ocorrência da infecção urinária 
na gravidez, o que poderá trazer benefícios para a saúde materna e fetal, visto que a identificação precoce desses fatores ajudará o profissional de saúde na prevenção de agravos e na tomada de decisão durante a assistência prestada no cuidado pré-natal.

Vale ressaltar que as complicações advindas da infecção urinária na gravidez contribuem com a alta taxa de mortalidade infantil no Brasil. Nesse sentido, esse estudo sugere o aprofundamento dessa temática através da realização de maior número de pesquisas científicas que possam elucidar outros fatores de risco.

\section{REFERÊNCIAS}

1. Oladeinde $\mathrm{BH}$, Omoregie R, Oladeinde OB. Asymptomatic Urinary Tract Infection among Pregnant Women Receiving Ante-Natal Care in a Traditional Birth Home in Benin City, Nigeria. Ethiop J Health Sci [Internet]. 2015 [acesso em 17 feb 2015];25(1):3 - 8. Available from: http://www.ncbi.nlm.nih. gov/pmc/articles/PMC4337079/

2. Kluczynik CEN, Silva DR, Sousa Neto JB de, Catão RMR. Occurrence of bacteriuria asymptomatic in pregnant women in a public maternity. Rev Enferm UFPE on line [Internet]. 2010 [acesso em 15 mar 2015];4(1):270-78. Available from: http://www.revista.ufpe.br/revistaenfermagem/index.php/ revista/article/view/773

3. Figueiró-Filho EA, Bispo AMB, Vasconcelos MM, Maia MZ, Celestino FG. Infecção do trato urinário na gravidez: aspectos atuais. Rev Femina [Internet]. 2009 [acesso em 20 maio 2015];37(3):165-71. Available from: http://www. febrasgo.og.br/site/wp-content/uploads/2013/05/Feminav37n3-p165.pdf

4. Ministério da Saúde. Mortalidade Infantil no Brasil: tendências, componentes e causas de morte no período de 2000 a 2010. Brasília (DF); 2011.

5. Soares CB, Hoga LAK, Peduzzi M, Sangaleti C, Yonekura T, Silva DRAD. Revisão integrativa: conceitos e métodos utilizados na enfermagem. Rev esc enferm USP [Internet]. 2014 [acesso em 24 abr 2015];48(2): 335-45. Available from: http://dx.doi. org/10.1590/S0080-6234201400002000020

6. Kysas PA. Evidence-Based Oral and Maxillofacial Surgery. J Oral Maxillofac Surg [Internet]. 2008 [acesso em 12 abr 2015];66 (3): 973-986. Available from: http://www.joms.org/ article/S0278-2391(08)00096-7/pdf

7. Hood PD. Scientific Research and Evidence-Based Practice. San Francisco: WestEd, 2003. 51p.

8. Rajaratnam A, Baby NM, Kuruvilla TS, Machado S. Diagnosis of asymptomatic bacteriruria and associated risk factors among pregnant women in Mangalore, Karnataka, India.J Clin Diagn Res [Internet]. 2014 [acesso em 20 abr 2015];8(9):235. Available from: http://www.ncbi.nlm.nih.gov/pmc/articles/ PMC4225942/

9. Vettore MV, Dias M, Vettore MV, Leal MC. Avaliação do manejo da infecção urinária no pré-natal em gestantes do Sistema Único de Saúde no município do Rio de Janeiro. Rev bras epidemiol [Internet]. 2013 [acesso em 15 abr 2015];16(2):33851. Available from: http://www.dx.doi.org/10.1590/S1415$790 \times 2013000200010$
10. Emiru T, Beyene G, Tsegaye W, Melaku S. Associated risk factors of urinary tract infection among pregnant women at FelegeHiwot Referral Hospital, Bahir Dar, North West Ethiopia, Etiópia. BMC Res Notes [Internet]. 2013 [acesso em 5 abr 2015];6(292). Available from: http://www.biomedcentral. com/1756-0500/6/292

11. Hackenhaar AA, Albernaz EP. Prevalência e fatores associados à internação hospitalar para tratamento da infecção do trato urinário durante a gestação. Rev Bras Ginecol Obstet [Internet]. 2013 [acesso em 24 abr 2015];35(5):199-204. Available from: http://febrasgo.tempsite.ws/rbgo/2013/5RBGOv35n5.pdf

12. Stokholm J, Schjorring S, Pedersen L, Bischoff AL, Folsgaard $\mathrm{N}$, Carson CG et al. Living with cat and dog increases vaginal colonization with $\mathrm{E}$. coli in pregnant women. Allergy, Asthma \& Clinical Immunology [Internet]. 2013 [acesso em 20 março 2015]; 9(15):1-9. Available from: http://www.aacijournal.com/ content/9/1/15

13. Hamdan ZH, Ziad AHM, Ali SK, Adam I. Epidemiology of urinary tract infections and antibiotics sensitivity among pregnant women at Khartoun North Hospital, Sudão. Ann Clin Microbiol Antimicrob [Internet]. 2011 [acesso em 14 abr 2015];10(2):[cerca 4 p.]. Available from: http://www.annclinmicrob.com/content/10/1/2

14. Haider G,Zehra N,Munir AA,HaiderA.Risk factors of urinary tract infection in pregnancy, Paquistão. J Pak Med Assoc [Internet]. 2010 [acesso em 01 abr 2015];60(3):[cerca de 4 p.]. Available from: http://www.ncbi.nlm.nih.gov/pubmed/20225781

15. Pagnonceli J, Abegg MA, Colacite J. Avaliação de infecção urinária em gestanes do município de Marechal Cândido Rondon - PR. Arq. Ciências saúde UNIPAR [Internet]. 2010 [acesso em 06 abr 2015];14(3):211-216. Available from: http://revistas.unipar.br/saude/article/viewFile/3662/2375

16. Amiri FN, Rooshan MH, Ahmady MH, Soliamani MJ. Hygiene practices and sexual activity associated with urinary tract infection in pregnant women, Iran. East Mediterr Health J [Internet]. 2009 [acesso em 16 abr 2015];15(1):10410. Available from: http://www.ncbi.nlm.nih.gov/ pubmed/19469432.

17. Ekachai Kovavisara MD, Maytina Vichaipruck MD, Suwattana Kanjarahareutai MSC. Risk factors related to asymptomatic bacteriuria in pregnant women. J Med Assoc Thai [Internet]. 2009 [acesso em 30 abr 2015]; 92 (5): 606-10. Available from: http://mat.or.th/journal/files/Nol92_No.5_606_9154.pdf

18. Nascimento WLS, Oliveira M, Araújo GLS. Infecção do trato urinário em gestantes usuárias do sistema único de saúde. Ensaios e C. [Internet]. 2012 [acesso em 12 abr 2015];16(4):111-123. Available from: http://www.redalyc.org/ articulo.oa?id=26029236009

19. Ebidor UL. Urinary tract infection amongst pregnant women in Amassoma, Southern Nigeria. Afr. J. Microbiol. Res. [Internet]. 2015 [acesso em 20 jun 2015];9(6):355-59. Available from: http://www.academicjournals.org/article/ article1424251765_Lawani\%20et\%20al.pdf

20. Onuoha SC, Fatokun K. Prevalence and antimicrobial susceptibility pattern of urinary tract infection (UTI) among pregnant women in Afikpo, Ebonyi, Nigeria. Am. J. Life Sci. [Internet] 2014 [acesso em 15 abr 2015];2(2):46-52. Available from: http://www.researchgate.net/publication/275567338 Prevalence_and_Antimicrobial_Susceptibility_Pattern_of_- 
Urinary_Tract_Infection(UTI)_among_Pregnant_Women_in_ Afikpo_Ebonyi_State_Nigeria

21. Okonko IO, ljandipe LA, Ilusanya AO, Donbrave-Emmanuel OB, EjembiJ,UdezeAO,Egun OC,FowotadeA,NkangAO.Incidence of urinary tract infection (UTI) among pregnant in Ibadan, SouthWestern Nigeria. Afr J Biotechnol [Internet]. 2009 [acesso em 24 jun 2015];8(23):6649-6657. Available from: http://www. ajol.info/index.php/ajb/article/view/66370/54082

22. Sharma JB, Shena A, saurabi SS, Kumar S, Roy KK. Prevalence of urinary incontinence and other urological problems during pregnancy. Arch Gynecol Obstet [Internet]. 2009 [acesso em 15 jun 2015];279(6):845-851. Available from: http://link. springer.com/article/10.1007\%2Fs00404-008-0831-0

23. Figueiredo A, Gomes G, Campos A. Infecções urinárias e gravidez - diagnóstico, terapêutica e prevenção. Acta Obstet Ginecol Port [Internet]. 2012 [acesso em 13 jan 2015];6(3):124-133. Available from: http://www.fspog.com/ fotos/editor2/1_ficheiro_608.pdf

24. Alvarez JR, Fechner AJ, Williams SF, Ganesh VL, Apuzzio J. Asymptomatic bacteriuria in pregestational diabetic pregnancies and the role of group B streptococcus. Am J Perinatol [Internet]. 2010 [acesso em 24 mar 2015];27(3):2314. Available from: https://www.thieme-connect.com/DOI/ DOI?10.1055/s-0029-1239485

25. Murray AC, Kuskowski MA, Johnson JR. Virulence factors predict Escherichia coli colonization patterns among human and animal household members. Ann. Intern. Med. [Internet] 2004 [acesso em 23 jan 2015];140(10):848-849. Available from: http://annals.org/article.aspx?articleid=717477

26. Johnson JR, Clabots C, Kuskowski MA. Multiple-host sharing, long-term persistence, and virulence of Escherichia coli clones from human and animal household members.J Clin Microbiol [Internet]. 2008 [acesso em 15 mar 2015]; 46(12):4078-4082. Available from: http://jcm.asm.org/content/46/12/4078.full. pdf+html

27. Moola S. Evidence-based practice. Online Brazilian Journal of Nursing [Internet] 2010 [acesso em agosto 2015];9(2).Available from: http://dx.doi.org/10.17665/1676-4285.2010v9n2 